Die Abteilung Daten, Demographie und Qualität (DDQ) erstellt Grundlagenpapiere basierend auf wissenschaftlicher Literatur zu verschiedenen Themen im Bereich Qualität, welche in der Schweizerischen Ärztezeitung veröffentlicht werden. Die FMH nimmt auf der Basis der erarbeiteten Grundlagen Stellung zum Thema und gelangt über das Papier «Die Meinung der FMH» mit ihrer Position an die Öffentlichkeit. Nachfolgend werden das Grundlagenpapier sowie die Meinung der FMH zum Thema «Shared Decision Making» präsentiert.

\title{
Shared Decision Making - Arzt und Patient entscheiden gemeinsam
}

\author{
Michelle Gerber, \\ Esther Kraft, \\ Christoph Bosshard
}

\footnotetext{
* Die Literatur findet sich unter www.saez.ch

$\rightarrow$ Aktuelle Ausgabe oder

$\rightarrow$ Archiv $\rightarrow 2014 \rightarrow 50$.
}

\section{Zusammenfassung}

Shared Decision Making (SDM) ist ein Modell der Entscheidungsfindung im klinischen Kontext, gemäss welchem Arzt ${ }^{1}$ und Patient aktiv Informationen austauschen, verschiedene Behandlungsoptionen abwägen und partnerschaftlich eine Entscheidung fällen. Entscheidend für das Gelingen von SDM ist, dass der Arzt während der ganzen Konsultation eine Atmosphäre schafft, in welcher sich Patienten frei äussern können. SDM wird in Situationen angewendet, in welchen einmalige Entscheidungen in Abhängigkeit der Präferenzen der Patienten getroffen werden, aber auch bei längerfristigen Interventionen zu Verhaltensänderungen. Dabei ist umstritten, ob SDM abgesehen von Notfällen immer durchgeführt werden soll oder bloss dann, wenn zwei gleichwertige Behandlungsoptionen vorliegen.

Wenn sogenannte Entscheidungshilfen eingesetzt werden, erhöht SDM das Wissen der Patienten über die Erkrankung, fördert deren aktiven Einbezug und hilft ihnen, Präferenzen zu reflektieren. Weiter gibt es Hinweise, dass mit SDM unnötige Behandlungen verhindert werden können. Gemäss bisheriger Evidenz hat SDM aber weder klar positive noch negative Auswirkungen auf Gesundheitszustand, Wohlbefinden und Zufriedenheit der Patienten. Insgesamt braucht es aber mehr und differenzierte Forschung zur Wirksamkeit von SDM.

Aus ethischen Gründen erscheint SDM sehr sinnvoll. Dennoch werden Patienten häufig nicht wie von ihnen gewünscht in die Entscheidungsfindung miteinbezogen. Entscheidend für die Umsetzung in die Praxis sind die Einstellungen und Kompetenzen der Ärzte in Bezug auf SDM. Hilfreich ist das Vorliegen von wirksamen Trainingsprogrammen und qualitativ hochwertigen Entscheidungshilfen.
\end{abstract}

Shared Decision Making (SDM) oder partizipative Entscheidungsfindung wird immer häufiger als ideales Modell der Entscheidungsfindung im klinischen Kontext gesehen. So hält der Zentralvorstand der FMH 2004 bei der Revision des Zivilgesetzbuches zum Erwachsenenschutz fest, dass Behandlungsentscheide bei urteilsunfähigen Patienten grundsätzlich im Konsens zwischen den Angehörigen und dem medizinischen Betreuungsteam gefällt werden sollen [1]*. SDM wird als einer der wichtigsten Paradigmenwechsel in der Medizin bezeichnet und häufig als ein Indikator von guter medizinischer Qualität bewertet [2-5]. Gemäss SDM-Modell treffen Arzt und Patient gemeinsam die Entscheidung für eine bestimmte Behandlung. In Abgrenzung zum vorherrschenden Paternalismus in der Arzt-PatientBeziehung früherer Generationen ist eine weniger autoritäre und mehr patientenzentrierte Arztrolle gefragt, welche sich in Ansätzen des «Patient Centered Care» und in verschiedenen patientenzentrierten Konzepten und Methoden wie beispielsweise Gesundheitskompetenz, Patientenempowerment oder Motivational Interviewing zeigt. Diese Entwicklungen zum stärkeren Einbezug von Patienten spiegeln sich in den aktuellen Diskussionen im Schweizer Gesundheitswesen. Der Bundesrat fordert im Bericht Gesundheit2020 zu seinen aktuellen gesundheitspolitischen Prioritäten, dass «die Patienten/-innen künftig eine vollwertige, gleichberechtigte und selbstbestimmte Rolle in der Beziehung zu den Gesundheitsfachpersonen erhalten» [6]. Im Kontrast zu der genannten Forderungen nach mehr Patientenautonomie wird in der aktuellen gesundheitspolitischen Diskussion aber auch vor einer Ökonomisierung der Medizin und einem Verständnis der Patienten als Kunden gewarnt [7].

In der Schweiz [8] bestehen - im Vergleich zu den USA [9] oder Deutschland, wo ein Förderschwerpunkt für Forschung zu SDM finanziert wird [10] kaum formale Initiativen zur Untersuchung, Verbreitung und Umsetzung von SDM. Neben ein paar Studien zum Thema SDM, wie beispielsweise eine

www.fmh.ch 
Bevölkerungsbefragung in Genf [11] oder eine Befragung von Assistenzärzten und ihren Weiterbildungsverantwortlichen [12], gibt es das Gesundheitscoaching [13] des Kollegiums für Hausarztmedizin (KHM), welches SDM miteinbezieht. In diesem Projekt unterstützen Ärzte ihre Patienten dabei, ihr Gesundheitsverhalten in einem selbstgewählten konkreten Bereich zu optimieren (weitere ProjektBeispiele siehe Cornuz et al. 2011 [8]). In der Schweiz wird SDM auch in Informationsbroschüren thematisiert: die von der Non-Profit-Organisation Dialog Ethik zur Arzt-Patient-Beziehung herausgegebene Patientenbroschüre [14] und der Leitfaden der SAMW zum Thema Kommunikation im medizinischen Alltag [15]. An einigen Universitäten werden Medizinstudierenden Inhalte aus dem Bereich SDM vermittelt und an Universitätskliniken Weiterbildungskurse durchgeführt [8].

\section{Was ist Shared Decision Making?}

Der Begriff SDM wird unterschiedlich verwendet und unklar von verwandten Konzepten abgegrenzt [3, 16-18]. Dieser Abschnitt zeigt auf, was unter SDM verstanden wird und wie sich SDM von anderen Modellen und Begriffen der Entscheidungsfindung im klinischen Kontext unterscheidet.

Die evidenzbasierte Medizin ist der Ausgangpunkt, um im klinischen Kontext Entscheidungen $\mathrm{zu}$ treffen, wenn mindestens zwei Optionen (inkl. der Möglichkeit des Abwartens) zur Auswahl stehen [2]. Beispielsweise muss entschieden werden, ob bei einer an Brustkrebs erkrankte Patientin eine Brust entfernt wird (Mastektomie) oder eine brusterhaltende Operation durchgeführt wird. Häufig sind die zur Auswahl stehenden Behandlungsoptionen im Prinzip gleichwertig (sogenannte «equipoise» [19]) mit verschiedenen Vor- und Nachteilen, die gegeneinander abgewogen werden müssen, wie z. B. längere Lebensdauer gegenüber verminderter Lebensqualität. Ausserdem sind die möglichen Auswirkungen einer Behandlung mit Unsicherheit behaftet und die Evidenz für Behandlungsempfehlungen nicht in jedem Fall stark. Da es grosse Unterschiede in den Präferenzen, Bedürfnissen und Werten gibt und $\mathrm{Pa}$ tienten über unterschiedliche Ressourcen im Umgang mit einer Erkrankung verfügen, können Ärztinnen und Ärzte gemäss dem SDM-Modell diese Entscheidung nur gemeinsam mit den Patienten treffen. Bei präferenzsensitiven Entscheidungen ist SDM daher besonders angebracht [3, 20-22]. Für die Umsetzung von SDM ist eine Grundfrage, in welchen Entscheidungssituationen SDM durchgeführt werden soll und wie die Auswahl der den Patienten zur Diskussion vorgelegten Behandlungsoptionen erfolgt [19, 20, 23]. Zum einen wird vorgeschlagen, dass SDM einzig angewandt wird, wenn gemäss medizinischer Evidenz zwei gleichwertige Optionen vorliegen, die keine Nachteile für die Bevölkerung haben und sich auch in Bezug auf die Kosten nur geringfügig unter- scheiden. Zum anderen wird zur Stärkung der Patientenautonomie gefordert, dass SDM immer durchgeführt wird, ausser wenn die Situation das nicht zulässt, z. B. wenn in einem Notfall schnell gehandelt werden muss.

Loh und Simon [24] definieren SDM als «ein Interaktionsprozess mit dem Ziel, unter gleichberechtigter aktiver Beteiligung von Patient und Arzt auf Basis geteilter Information zu einer gemeinsam verantworteten Übereinkunft zu kommen». SDM zeichnet sich durch folgende Merkmale [3] aus:

- Involviert sind mindestens zwei Beteiligte (ein Arzt und ein Patient), häufig aber noch weitere Personen wie z.B. Familie, Freunde und weitere Gesundheitsfachpersonen.

- Beide Beteiligte nehmen aktiv am Prozess der Entscheidungsfindung teil.

- Zwischen beiden Beteiligten findet ein Informationsaustausch statt.

- Beide Parteien wollen gemeinsam eine Behandlungsentscheidung treffen.

SDM unterscheidet sich von anderen theoretischen Modellen der klinischen Entscheidungsfindung (Tabelle 1) am meisten darin, dass Arzt und Patient in allen Phasen der Entscheidungsfindung (Informationsaustausch, Abwägungsprozess und Treffen der Entscheidung) gemeinsam beteiligt sind. Bei den anderen drei Modellen verläuft der Informationsaustausch einseitig. Im paternalistischen und im interpretativen Modell wägt der Arzt alleine die Vor- und Nachteile verschiedener Behandlungsmöglichkeiten $\mathrm{ab}$ und trifft anschliessend die Entscheidung. Im Informed Decision Modell führt der Patient diese Schritte alleine durch. Ein weiterer Unterscheidungspunkt der Modelle bezieht sich auf die Arztrolle und auf das Patientenbild. Gemäss dem SDMModell sind Patient und Arzt gleichberechtigte, sich ergänzende Partner in der Entscheidungsfindung. Patienten bringen Informationen bezüglich ihres Lebensumfeldes, ihrer Werte, Bedürfnisse und Ängste sowie ihr subjektives Wissen über ihre Gesundheit und die Erkrankung in die Diskussion ein. Der Arzt vermittelt fachliches Wissen und klinische Erfahrung, aber auch ethische Werte, und trägt durch eine objektive Distanz zur klinischen Problematik zu einer optimalen Entscheidungsfindung bei [24, 25]. Aus Patientensicht ist eine der meistgenannten Barrieren für SDM das Machtungleichgewicht zwischen Arzt und Patient. Patienten möchten keine unangenehmen Patienten sein und die beschäftigten Ärztinnen und Ärzte nicht stören [27]. Für die Durchführung von SDM ist deshalb nicht nur der Moment der Entscheidung wichtig, sondern auch die Atmosphäre und Interaktion während der gesamten Konsultation. Diese ist geprägt durch folgende Faktoren: Sensibilität für die Bedürfnisse der Patienten, Einfühlungsvermögen, individualisierte Informationen, Ermutigung von Patienten, sich aktiv zu beteiligen, 
Tabelle 1

Modelle der Entscheidungsfindung im klinischen Kontext. ${ }^{1}$

\begin{tabular}{|c|c|c|c|c|}
\hline & Paternalistisches Modell & Interpretatives Modell2 & $\begin{array}{l}\text { Shared Decision Making } \\
\text { Modell }\end{array}$ & Informed Decision Modell \\
\hline Arztrolle & Beschützer, Wohltäter & Stellvertreter des Patienten & Partner & Technischer Experte \\
\hline Informationsaustausch & $\begin{array}{l}\text { Einseitig } \\
\text { Arzt } \rightarrow \text { Patient }\end{array}$ & $\begin{array}{l}\text { Einseitig } \\
\text { Arzt } \rightarrow \text { Patient (Wissen) } \\
\text { Patient } \rightarrow \text { Arzt (Werte) }\end{array}$ & $\begin{array}{l}\text { Gegenseitig } \\
\text { Arzt } \leftrightarrow \text { Patient }\end{array}$ & $\begin{array}{l}\text { Einseitig } \\
\text { Arzt } \rightarrow \text { Patient }\end{array}$ \\
\hline $\begin{array}{l}\text { Einbezug von Werten und } \\
\text { Präferenzen des Patienten }\end{array}$ & $\begin{array}{l}\text { Präferenzen sind nicht } \\
\text { relevant für eine objektive } \\
\text { Entscheidung. }\end{array}$ & $\begin{array}{l}\text { Arzt erfragt Präferenzen vom } \\
\text { Patienten und bezieht sie in } \\
\text { den Abwägungsprozess mit ein. }\end{array}$ & $\begin{array}{l}\text { Arzt diskutiert Präferenzen mit } \\
\text { dem Patienten. }\end{array}$ & $\begin{array}{l}\text { Patient kennt die eigenen } \\
\text { Präferenzen. }\end{array}$ \\
\hline Abwägungsprozess & \multicolumn{2}{|c|}{ Arzt alleine, evtl. gemeinsam mit anderen Fachpersonen. } & $\begin{array}{l}\text { Arzt und Patient gemeinsam, } \\
\text { evtl. mit sozialem Umfeld und } \\
\text { anderen Fachpersonen. }\end{array}$ & $\begin{array}{l}\text { Patient alleine, evtl. mit } \\
\text { sozialem Umfeld und anderen } \\
\text { Fachpersonen. }\end{array}$ \\
\hline Entscheidung treffen & \multicolumn{2}{|l|}{ Arzt, Patient gibt Zustimmung } & Arzt und Patient gemeinsam & Patient \\
\hline Ärztliche Aufgabe & $\begin{array}{l}\text { Basierend auf Fachwissen und } \\
\text { Erfahrung ist der Arzt ethisch } \\
\text { verpflichtet, die objektiv beste } \\
\text { Behandlung für den Patienten } \\
\text { zu wählen und sich dessen } \\
\text { Zustimmung zu holen. }\end{array}$ & $\begin{array}{l}\text { Basierend auf Fachwissen, } \\
\text { Erfahrung und den erfragten } \\
\text { Werten des Patienten ist der } \\
\text { Arzt ethisch verpflichtet, die } \\
\text { optimale Behandlung für den } \\
\text { Patienten zu wählen und sich } \\
\text { dessen Zustimmung zu holen. }\end{array}$ & $\begin{array}{l}\text { Basierend auf Fachwissen und } \\
\text { Erfahrung informiert der Arzt } \\
\text { den Patienten über verschie- } \\
\text { dene Behandlungsmöglich- } \\
\text { keiten und erfragt die Präferen- } \\
\text { zen des Patienten. } \\
\text { Der Arzt ist ethisch verpflichtet, } \\
\text { den Patienten zu ermutigen, am } \\
\text { Entscheidungsprozess teilzuneh- } \\
\text { men, ihn dabei zu unterstützen } \\
\text { und gemeinsam die optimale } \\
\text { Behandlung zu wählen. }\end{array}$ & $\begin{array}{l}\text { Basierend auf Fachwissen und } \\
\text { Erfahrung ist der Arzt ethisch } \\
\text { verpflichtet, den Patienten } \\
\text { neutral und verständlich über } \\
\text { verschiedene Behandlungs- } \\
\text { möglichkeiten zu informieren, } \\
\text { damit dieser unbeeinflusst } \\
\text { die beste Behandlung wählen } \\
\text { kann. }\end{array}$ \\
\hline Patientenbild & \multicolumn{2}{|c|}{$\begin{array}{l}\text { Patient kann aufgrund mangelndem Wissens, } \\
\text { mangelnder Objektivität und Einschränkungen durch die } \\
\text { Erkrankung keine optimale Entscheidung treffen. }\end{array}$} & $\begin{array}{l}\text { Patient kann gemeinsam mit } \\
\text { dem Arzt entscheiden, wenn } \\
\text { er dazu ermutigt und im Ent- } \\
\text { scheidungsprozess begleitet } \\
\text { wird. }\end{array}$ & $\begin{array}{l}\text { Patient kann selbstständig ent- } \\
\text { scheiden, wenn ihm fehlendes } \\
\text { Wissen auf verständliche Weise } \\
\text { vermittelt wird. }\end{array}$ \\
\hline
\end{tabular}

Basierend auf: [16, 23, 26].

Diese vier Modelle werden häufig in der wissenschaftlichen Literatur genannt. Daneben sind weitere Modelle denkbar, z. B. ein Coaching-Modell, in welchem der Arzt gemeinsam mit den Patienten Informationen austauscht und den Abwägungsprozess durchführt, aber den Patienten anleitet, die Entscheidung selber zu treffen.

2 Auch Professional-as-agent-Modell genannt.

sowie Möglichkeiten für Patienten, ihren Gedanken und Emotionen Ausdruck zu verleihen. So beteiligen sich Patienten aktiver, äussern ihre Präferenzen und Werte, benennen eher Barrieren der Behandlungen oder berichten von nicht eingehaltenen Behandlungsentscheidungen wie zum Beispiel unregelmässige Medikamenteneinnahmen. Eine gute Beziehung ist auch deshalb relevant, da die Konsultation beim Arzt für sich bereits eine therapeutische Intervention ist [28-31].

Verwandt mit diesen Modellen der klinischen Entscheidungsfindung ist der Begriff «informed consent» oder «informierte Zustimmung». Mit diesem wird die rechtliche Autorisation von Patienten zur Durchführung einer Behandlung bezeichnet, welche an gewisse Bedingungen wie die Urteilsfähigkeit des Patienten und verständliche Aufklärung zu Risiken und Chancen einer Behandlung geknüpft ist. Der Arzt schlägt eine Behandlung vor und informiert über allfällige alternative Behandlungsmöglichkeiten, aus welchen der Patient auswählen kann. Dabei wird die Zustimmung des Patienten zur
Durchführung der Behandlung eingeholt [3, 23, 26]. Wichtig ist, dass Ärztinnen und Ärzte ihrer Aufklärungspflicht, wie sie in den kantonalen Gesundheitsgesetzten verankert ist, korrekt nachkommen und dies bei Bedarf auch nachweisen können [3234]. Wenn sie unter diesen Bedingungen eine heikle Behandlungsentscheidung treffen, in welcher die Lebensqualität höher gewichtet wird als die Lebensdauer, brauchen sie keine Haftpflichtansprüche oder Schadenersatzforderungen zu fürchten.

\section{Prozess des Shared Decision Making}

Um die Umsetzung des SDM in der klinischen Praxis zu erleichtern, haben Charles et al. [16] und Elwyn et al. [25] Prozessmodelle entwickelt. Diese Modelle unterscheiden vier Phasen des SDM: Einführung, Informationsaustausch, Abwägungsprozess, Entscheidung.

\section{Einführung}

In dieser Phase wird den Patienten vermittelt, was das medizinische Problem ist und welche Behand- 
lungsmöglichkeiten es gibt - inklusive der Möglichkeit abzuwarten und zu beobachten. Ausserdem erklärt der Arzt den Patienten, wie die Entscheidungsfindung abläuft und welche Rollen beide haben. Dabei vermittelt er ihnen, dass die Entscheidung gemeinsam getroffen wird, so dass er sich mit der schwierigen Entscheidung nicht allein gelassen fühlt $[25,35]$.

\section{Informationsaustausch}

In einer nächsten Phase informiert der Arzt über die Erkrankung, die Behandlungsoptionen, die dazugehörigen Risiken und Nutzen in Bezug auf die Gesundheit, das physische und psychische Wohlbefinden und die sozialen Lebensumstände der Patienten. Er bringt auf diese Weise medizinische Evidenz in die klinische Konsultation mit ein und überprüft gleichzeitig, ob die Patienten alles richtig verstanden haben. Dabei werden Befürchtungen und subjektive Krankheitstheorien der Patienten sowie allfällige Vorschläge für weitere Optionen miteinbezogen. Patienten bringen ausserdem ihr Wissen zu ihrer Person in die Konsultation ein wie beispielsweise ihre Krankengeschichte, Lebenssituation und Werte [16, 17, 21, 25]. Der Arzt unterstützt Patienten, sich mittels Broschüren oder geeigneter Webseiten selbst Wissen anzueignen und dieses zu interpretieren [5]. Zusätzlich werden in dieser Phase auch sogenannte Decision Aids (Entscheidungshilfen) verwendet. Diese Broschüren, Videos oder webbasierte Programme informieren Patienten in standardisierter Weise über die Erkrankung und die Behandlungsoptionen mit den dazugehörigen Risiken, stellen Wahrscheinlichkeiten verständlich dar und unterstützen Patienten, ihre Präferenzen herauszufinden [35-37]. Auch weitere Hilfsmittel wie graphische Darstellungen von Wahrscheinlichkeiten oder von Optionen mit ihren Vor- und Nachteilen (Option grids) können hilfreich sein [21]. Diese verschiedenen Hilfsmittel dienen da$\mathrm{zu}$, den Informationsaustausch (und Abwägungsprozess) systematisch und standardisiert durchzuführen und fördern den aktiven Einbezug von Patienten. Sie sind aber kein zwingender Bestandteil eines SDMProzesses und ihr Einsatz garantiert noch keine Entscheidungsfindung gemäss dem SDM-Modell.

\section{Abwägungsprozess}

Nach der gemeinsamen Informationsaustauschphase erfolgt ein Abwägungsprozess der verschiedenen Vor- und Nachteile der zur Wahl stehenden Behandlungsoptionen. Dazu erfragt der Arzt die Erwartungen, Werte, Sorgen und Ideen der Patienten und unterstützt sie darin, die eigenen Präferenzen herauszufinden und zu gewichten. Die Entscheidung für eine Behandlungsoption wird auch von der Wahrscheinlichkeit der verschiedenen Auswirkungen einer Behandlung und der Selbstwirksamkeitserwartung der Patienten beeinflusst. Mit Letzterem ist gemeint, ob sich Patienten zutrauen, nötige Behand- lungsschritte (z. B. regelmässige Medikamenteneinnahme) erfolgreich durchzuführen. Viele Patienten wollen die Meinung ihres sozialen Umfeldes in den Entscheidungsprozess miteinbeziehen und die Meinung von weiteren beteiligten Ärzten und Gesundheitsfachpersonen hören. Im Gegensatz zum Informed Decision Modell beschränkt sich die Rolle des Arztes nicht auf das neutrale Vermitteln von Fachwissen. Der Arzt legt ebenfalls die eigene Sichtweise zu den verschiedenen Optionen dar und kann Empfehlungen geben. Dies bedeutet aber nicht, dass Ärztinnen und Ärzte einfach versuchen, Patienten von ihrer bereits getroffenen Entscheidung zu überzeugen (vgl. Karnieli-Miller und Eisikovits [38]). Da zwischen den Beteiligten ein Machtungleichgewicht besteht, muss für Patienten eine Atmosphäre geschaffen werden, in welcher sie sich sicher fühlen und frei äussern können [16, 17, 25, 39].

\section{Behandlungsentscheidung fällen}

Viele Patienten brauchen Zeit, um sich die Entscheidung zu überlegen, weshalb Entscheidungen verschoben und Folgekonsultationen vereinbart werden. Gemäss dem SDM-Modell treffen Arzt und Patient gemeinsam eine Entscheidung. Es kann aber vorkommen, dass sich die Beteiligten trotz gemeinsamer Diskussion nicht einig werden. Wenn der Arzt die bevorzugte Behandlungsoption des Patienten nicht umsetzen will, kann sich dieser an einen anderen Arzt wenden. Umgekehrt hat der Patient jederzeit das Recht, eine bestimmte Behandlungsoption abzulehnen. Das zeigt, dass beide Beteiligte Einschränkungen unterworfen sind und nicht eine Person alleine entscheiden kann [16, 17, 25].

Die Entscheidungsfindung im klinischen Kontext ist ein dynamischer Prozess - die beschriebenen Phasen gehen fliessend ineinander über. Innerhalb einer Konsultationsphase kann sich das Entscheidungsfindungsmodell (Tabelle 1) ändern oder Elemente verschiedener Modelle werden kombiniert. Beispielsweise beginnt ein Arzt eine Konsultation mit dem SDM-Modell, wechselt aber schliesslich zum Interpretativen Modell, wenn der Patient wünscht, dass der Arzt die Behandlungsentscheidung für ihn trifft. Die Anwendung der verschiedenen Modelle der Entscheidungsfindung wird von der klinischen Situation und den Bedürfnissen des Patienten beeinflusst und erfordert deshalb Flexibilität [16].

\section{Anwendung in verschiedenen Fachbereichen} Die bekannteste Definition des SDM-Modells [3] wurde für den Kontext einer potenziell lebensbedrohlichen Krankheit wie etwa eine Krebserkrankung entwickelt, für welche es mehrere Behandlungsalternativen mit verschiedenen möglichen, mit Unsicherheit behafteten Folgen gibt (vgl. Anwendung in der Onkologie: Politi et al. 2012 [40]). Das SDM-Modell wird auch in anderen Bereichen angewendet, z. B. in der Intensivmedizin bei Ent- 
scheidungen zu lebenserhaltenden Massnahmen [41], in der Rehabilitation [42] oder in der Psychiatrie [43].

Das Modell von Charles et al. [3] aus dem Bereich der Onkologie wurde für den Kontext der medizinischen Grundversorgung [5] und der Behandlung von chronisch kranken Patientinnen und Patienten [31] erweitert. Gemäss Murray und Kollegen [5] zeichnet sich die Konsultation in der Grundversorgung durch undifferenzierte Symptome und teilweise durch multiple Diagnosen und Probleme aus, welche auch durch psychische und soziale Faktoren beeinflusst werden. Im Modell von Charles et al. [3] hingegen liegt eine klare Diagnose vor, welche beiden Parteien bekannt ist, bevor SDM angewendet wird. Für die Praxis der medizinischen Grundversorgung ist es deshalb wichtig, dass SDM bereits zu Beginn der Konsultation bei der Auftragsklärung beginnt. Arzt und Patient müssen sich darauf einigen, welche Probleme besprochen werden, ob sie jetzt oder zu einem späteren Zeitpunkt besprochen werden und ob der Arzt überhaupt die richtige Ansprechperson ist. In der medizinischen Grundversorgung müssen häufig mehrere Entscheidungen getroffen werden, die aber weniger akute Auswirkungen auf die Patienten haben und laufend revidiert werden können. Wichtig ist deshalb auch eine gemeinsame Evaluation von Entscheidungen.

Im Gegensatz zu einer akuten Erkrankung wie im Modell von Charles et al. [3] ist bei der Behandlung von chronisch kranken Patienten oder in der Gesundheitsförderung und Prävention die Rolle des Arztes weniger auf das Vermitteln von Expertenwissen als auf die Umsetzung einer Entscheidung ausgerichtet. Elwyn und Kollegen [19, 44] sprechen deshalb von Interventionen zur Verhaltensänderung in Abgrenzung zu Interventionen zur Entscheidungsunterstützung. Ein Beispiel für eine Behandlungsentscheidung zur Verhaltensänderung ist, ob ein an Diabetes erkrankter Patient regelmässig Medikamente einnimmt oder seine Essgewohnheiten ändert. Da sich in diesen Interventionen die Konsequenzen der getroffenen Entscheidung häufig erst längerfristig zeigen, macht dies die Umsetzung einer Entscheidung zu einer Herausforderung. Deshalb ist es besonders wichtig, dass die Selbstwirksamkeit der Patienten zur Umsetzung von Behandlungsmassnahmen und die Unterstützung durch ihr soziales Umfeld in die Entscheidung miteinbezogen werden sowie die Patienten die Entscheidung mittragen (siehe auch Motivational Interviewing [44]). Bei der Behandlung chronisch kranker Patienten besteht häufig eine langfristige Beziehung zwischen Patient und Arzt, respektive Patient und weiteren wichtigen Gesundheitsfachpersonen. Dies ist eine ideale Voraussetzung für eine der wichtigsten Grundlagen für SDM: eine Partnerschaft zwischen Arzt und Patient $[5,31,42]$.

\section{Warum Shared Decision Making?}

In der Einleitung wurde festgestellt, dass SDM als ideales Modell der klinischen Entscheidungsfindung gilt. Im Folgenden sollen die Gründe für diese Position aufgeführt und mit wissenschaftlichen Ergebnissen und Diskussionspunkten ergänzt werden.

\section{Bedürfnis der Patienten}

Patienten sind im Vergleich zu früher skeptischer gegenüber Ärztinnen und Ärzten und sind sich ihrer Rechte als Patienten bewusster. Viele Patienten informieren sich über das Internet zu Gesundheitsthemen und verschiedenen Behandlungsmöglichkeiten und wollen demzufolge auch bei medizinischen Entscheidungen mitreden [20, 22]. Befragungen zeigen, dass sich eine Mehrheit an medizinischen Entscheidungen beteiligen möchte. Andererseits gibt es auch eine Minderheit, welche die Entscheidung lieber dem Arzt überlassen möchte [26, 45]. So gaben in einer Bevölkerungsbefragung in Deutschland 57\% der Personen an, dass medizinische Entscheidungen gemeinsam mit dem Arzt getroffen werden sollten, für weitere $17 \%$ sollten Patienten alleine entscheiden und nur $23 \%$ meinten, der Arzt solle alleine entscheiden [46]. Dabei hat die Präferenz für eine aktive Beteiligung an Entscheidungen in den 2000er-Jahren zugenommen [45]. Der Wunsch nach einer Beteiligung an medizinischen Entscheidungen hängt vom Land respektive von der Kultur [47], der Patientengruppe [11, 20, 45, 46], der Befragungsmethode $[22,45]$ sowie der Frageformulierung und Definition [45] ab. So möchten fast alle Patienten mehr Informationen zur Erkrankung und zu den Behandlungsmöglichkeiten, aber nur ein Teil möchte sich an der Entscheidung beteiligen. An der Diagnosestellung und Festlegung der Behandlungsalternativen möchte sich dagegen kaum jemand beteiligen [20, 22, 26]. Eine aktive Involvierung wird häufiger von Frauen, Personen mit einem höheren Bildungsabschluss, aus einer höheren sozioökonomischen Schicht, von Inländern und von jüngeren Personen gewünscht, wobei soziodemographische Angaben im Einzelfall keine zuverlässige Indikatoren für Partizipationspräferenzen sind [11, 46]. Insgesamt werden nur rund $60 \%$ der Patienten ihren Wünschen entsprechend in die Entscheidungsfindung einbezogen [4]; SDM wird in der Praxis eher selten angewandt [48]. Viele Ärztinnen und Ärzte unterschätzen vermutlich das Bedürfnis nach Informationen und Mitentscheidung: Sie handeln häufig gemäss den von ihnen wahrgenommenen Erwartungen der Patienten anstatt die Präferenzen direkt zu erfragen [22, 26, 49].

\section{Ethische Überlegungen}

Indem die Autorisation von Patienten zur Durchführung einer Behandlung (informed consent) ethisch und rechtlich als Patientenrecht etabliert ist, wird auch ein Minimum an SDM nötig. Ausserdem erhalten Patienten durch SDM mehr Informationen, ihre 
Autonomie wird gestärkt und sie erhalten ein Gefühl der Kontrolle über ihre Behandlung. Mit SDM werden die Patienten aber nicht mit der Entscheidung alleine gelassen, sondern der Arzt ist verpflichtet, seine Expertise einzubringen und sie im Entscheidungsprozess zu begleiten. Aus einer ethischen Sichtweise wird deshalb eine geteilte Entscheidung für wünschenswert angesehen [3, 22].

\section{Auswirkung auf die Gesundheit}

Es wird angenommen, dass SDM durch das systematische Abwägen aller Optionen die medizinische Qualität erhöht, unnötige Behandlungen verhindert, bei Patienten mit chronischen Krankheiten für sich genommen therapeutisch wirkt sowie die Adhärenz (Compliance oder Therapietreue) erhöht und so positive Auswirkungen auf den Gesundheitszustand der Patienten hat $[20,35,39,50]$. Andererseits ist durch SDM nicht ein allgemein besserer Gesundheitszustand zu erwarten. Patienten erreichen aber eher die gewünschten Behandlungsergebnisse bzw. diejenigen Ergebnisse treten nicht ein, welche sie vermeiden wollten [37]. In der Tat weisen Studien keine klare Verbesserung des Gesundheitszustandes und der allgemeinen Lebensqualität nach [37, 50-52]. Tendenziell finden sich eher Effekte sowohl für Studien mit chronisch kranken Patienten, in welchen eine längerfristig Arzt-Patient-Beziehung besteht, als auch in Situationen, in welchen SDM während mehreren Konsultationen stattfand und ein Training des Arztes in SDM durchgeführt wurde [51]. Gemäss der aktuellen Evidenz hat SDM also weder klar positive noch negative Auswirkungen auf den Gesundheitszustand. Gesicherte Aussagen zur Wirksamkeit von SDM können allerdings nur mit Vorsicht gemacht werden. Denn es gibt nur eine geringe Anzahl von qualitativ hochwertigen Studien und in diesen werden verschiedene Arten von SDM-Interventionen bei sehr unterschiedlichen Patientengruppen analysiert. Ausserdem fehlt in den Studien häufig eine Kontrolle der Qualität der Implementierung von SDM.

\section{Aktuelle}

Forumthemen

Diskutieren Sie mit! Im Forum präsentieren wir regelmässig brisante Themen aus Politik, Ökonomie und Wissenschaft, die das Schweizer Gesundheitswesen betreffen. Bringen Sie Ihre Meinung ein oder kommentieren Sie die Äusserungen Ihrer Kolleginnen und Kollegen. Das Forum finden Sie unter: www.saez.ch/forum/

\section{Auswirkung auf die Beteiligung} und Zufriedenheit

SDM erhöht nachweislich das Wissen der Patienten über ihre Erkrankung, insbesondere wenn Entscheidungshilfen verwendet werden $[37,50,51]$. Wenn Entscheidungshilfen verwendet werden, sind sich die Patienten über ihre eigenen Werte klarer und fühlen sich seltener unentschlossen. Mehr Arzt-PatientKommunikation über die Entscheidung findet statt, und die Patienten sind aktiver. Allerdings entscheiden die Patienten in Studien zu Entscheidungshilfen häufiger alleine (Informed Decision Modell), während SDM nicht häufiger stattfindet [37]. Ob SDM wie vermutet die Adhärenz erhöhen kann, ist aufgrund der geringen Anzahl von Studien unklar [37, 50, 51]. In Bezug auf die Zufriedenheit sind die Ergebnisse ebenfalls unklar und deuten höchstens auf einen kleinen Effekt hin [37, 50, 51].

\section{Auswirkung auf das Gesundheitssystem}

Nachgewiesen wurden regionale Unterschiede in der Häufigkeiten von Behandlungen innerhalb eines Landes, die nicht durch die Krankheit oder die Präferenzen der Patienten erklärt werden können, sondern vermutlich durch sich unterscheidende lokale ärztliche Meinungen [53]. Solche Unterschiede in der Behandlung könnten durch den konsequenten Einbezug der Präferenzen von Patienten durch SDM vermindert werden. Es gibt Hinweise, dass SDM unnötige Behandlungen verhindert, welche keinen Nutzen oder beträchtliche Risiken und Nebenwirkungen mit sich bringen [21]. Durch den Einsatz von Entscheidungshilfen werden häufiger konservative Behandlungen anstatt grössere Operationen gewählt [37]. Nach einem Training von Hausärzten in SDM setzten diese seltener Antibiotika für die Behandlung von Patienten mit akuten respiratorischen Infektionen ein [54].

Häufig wird befürchtet, dass durch SDM die Konsultationszeit zunimmt und damit die Kosten für Gesundheitsleistungen steigen. Mangelnde Zeit ist in der Tat das von Ärzten am häufigsten genannte Hindernis für die Umsetzung von SDM [16, 22, 49, 55] und wird auch von Patienten als Barriere für SDM wahrgenommen [27]. Zwei Cochrane Reviews [37, 50] zeigen allerdings, dass die Konsultationen mit durchschnittlich nur drei Minuten mehr kaum länger dauern. Dennoch sind eher positive Auswirkungen von SDM zu erwarten, wenn mehr Zeit für die Konsultationen zur Verfügung steht [56].

Ob SDM zu einer Reduktion der Gesundheitskosten beitragen kann oder diese im Gegenteil weiter erhöht, kann aufgrund kaum vorhandener Studien nicht beantwortet werden. Die wenigen Studien weisen darauf hin, dass SDM vermutlich keinen grossen Einfluss auf die Kosten hat [37].

\section{Schlussfolgerungen}

SDM ist kein Wundermittel. Wenn sogenannte Entscheidungshilfen eingesetzt werden, erhöht es das Wissen der Patienten über die Erkrankung, fördert deren aktiven Einbezug und hilft ihnen, Präferenzen zu reflektieren. Es gibt Hinweise, dass durch SDM unnötige Behandlungen verhindern werden können. SDM hat aber gemäss der aktuellen Evidenz weder klar positive noch negative Auswirkungen auf Gesundheitszustand, Wohlbefinden und Zufriedenheit der Patienten und erhöht laut Studien die Konsultationszeit nicht wesentlich. Viele Fragen zu seiner Wirksamkeit bleiben aber noch offen. Es gibt zwar sehr viele Studien zu Entscheidungshilfen, aber nur wenige zu SDM. Dabei bleibt unklar, ob eine grössere Wirksamkeit bei einer hohen Qualität der Implementierung oder für bestimmte Patientengruppen erreicht werden kann. 
Ob SDM umgesetzt wird, hängt auch von den Einstellungen und Erwartungen der Ärztinnen und Ärzte ab. Ein Wissensaustausch alleine reicht nicht aus, sondern es ist auch nötig, den Patienten zu einer Beteiligung zu ermutigen und eine gemeinsame Entscheidung treffen zu wollen. Eine Barriere für die Umsetzung von SDM kann die Angst der Ärzte vor möglichen Haftpflichtansprüchen oder Schadenersatzforderungen sein, wenn ein Patient etwa die Lebensqualität höher gewichtet als die Lebensdauer. Wichtig ist deshalb auch bei einer gemeinsamen Entscheidung, dass die Ärzte ihre Patienten nachweisbar korrekt aufklären. Fehlende Kompetenzen, Wissen und Vorbilder in Bezug auf SDM bezeichnen Ärzte häufig als weitere Hürde [49] - diese erwiesen sich in einer Studie mit Ernährungsberatern gar als die entscheidenden Faktoren [55]. Obwohl es zahlreiche SDM-Trainingsprogramme in verschiedenen Ländern gibt, unter anderem auch der Schweiz [57], fehlen Forschungsergebnisse, welche Art von Trainingsprogrammen effektiv ist. Zuletzt ist auch das Fehlen von evidenzbasierten und verständlichen Informationen und Entscheidungshilfen von hoher Qualität in vielen Bereichen hinderlich [22, 27, 49]. Eine Übersicht über bestehende Entscheidungshilfen und Hinweise für die Beurteilung von deren Qualität findet sich bei Lenz et al. 2012 [36] und Stacey et al. 2014 [37]. Hilfreich für die Umsetzung von SDM ist ausserdem, wenn unterschiedliche
Patientenpräferenzen in Guidelines explizit mit verschiedenen Optionen verknüpft werden. Damit SDM überhaupt möglich ist, dürfen Ärztinnen und Ärzten nicht so enge Vorgaben gemacht werden, dass diese unterschiedliche Patientenpräferenzen nicht mehr berücksichtigen können [2,5].

Aus ethischen Gründen erscheint SDM ein sehr sinnvolles Modell der klinischen Entscheidungsfindung zu sein. Unumstritten ist das Modell bei präferenzsensitiven Entscheidungen, für welche mehrere, aus ärztlicher Sicht gleichwertige Optionen vorhanden sind. SDM ermöglicht die von vielen Patienten gewünschte aktive Beteiligung, ohne dass die Patienten dabei allein gelassen und überfordert sind. Wichtig ist dabei, dass SDM flexibel gehandhabt wird, da sich die Bedürfnisse der Patienten mit der Zeit ändern können und sich von Patient zu Patient unterscheiden. Wie stark Patienten in die Entscheidung einbezogen werden, sollte an deren Bedürfnis angepasst werden.

\section{Interaktiver Artikel}

Wollen Sie diesen Artikel kommentieren? Nutzen Sie dafür die Kommentarfunktion in der OnlineVersion oder sehen Sie nach, was Ihre Kolleginnen und Kollegen bereits geschrieben haben:

www.saez.ch/aktuelle-ausgabe/interaktive-beitraege/

\title{
Wissen, was läuft.
} Das News-Paket der FMH.

\author{
Schweizerische Ärztezeitung, Today's Press, \\ FMH-Flash. Für Mitglieder kostenlos.
}




\section{Literatur}

1 Brunner HH, Steiner U. Revision Zivilgesetzbuch: Erwachsenenschutz / Vorentwurf Bundesgesetz über das Verfahren vor den Kindes- und Erwachsenenschutzbehörden. Stellungnahme. Bern: FMH; 2004.

2 Barratt A. Evidence Based Medicine and Shared Decision Making: The challenge of getting both evidence and preferences into health care. Patient Educ Couns. 2008;73:407-412.

3 Charles C, Gafni A, Whelan T. Shared decision-making in the medical encounter: What does it mean? (Or it takes at least two to tango). Social Science \& Medicine. 1997;44:681-692.

4 Kiesler DJ, Auerbach SM. Optimal matches of patient preferences for information, decision-making and interpersonal behavior: evidence, models and interventions. Patient Educ Couns. 2006;61:319-41.

5 Murray E, Charles C, Gafni A. Shared decision-making in primary care: tailoring the Charles et al. model to fit the context of general practice. Patient Educ Couns. 2006;62:205-11

6 Bundesamt für Gesundheit (BAG). Gesundheit2020 23.1.2013. Available from: http://www.bag.admin.ch/ gesundheit2020/index.html?lang=de.

7 Ziltener E. Patientin oder Kundin? - eine existentielle Frage. Bulletin der Schweizerischen Gesellschaft für Soziologie. 2012;142:37-39.

8 Cornuz J, Kuenzi B, Krones T. Shared decision making development in Switzerland: room for improvement! Z Evid Fortbild Qual Gesundhwes. 2011;105:296-9.

9 Frosch DL, Moulton BW, Wexler RM, Holmes-Rovner M, Volk RJ, Levin CA. Shared decision making in the United States: policy and implementation activity on multiple fronts. Z Evid Fortbild Qual Gesundhwes. 2011;105:305-12.

10 Härter M, Muller H, Dirmaier J, Donner-Banzhoff N, Bieber C, Eich W. Patient participation and shared decision making in Germany - history, agents and current transfer to practice. Z Evid Fortbild Qual Gesundhwes. 2011;105:263-70.

11 Cullati S, Courvoisier DS, Charvet-Berard AI, Perneger TV. Desire for autonomy in health care decisions: a general population survey. Patient Educ Couns. 2011;83:134-8.

12 Van der Horst K, Giger M, Siegrist M. Attitudes toward shared decision-making and risk communication practices in residents and their teachers. Medical Teacher. 2011;33:e358-e363.

13 Neuner-Jehle S, Schmid M, Grüninger U. The "Health Coaching" programme: a new patient-centred and visually supported approach for health behaviour change in primary care. BMC Fam Pract. 2013;14:100.

14 Kalbermatten-Casarotti P, Baumann-Hölzle R, Ritzenthaler-Spielmann D. Arzt-Patienten-Partnerschaft. Gemeinsam zum stimmigen Entscheid. Informationen für Patientinnen und Patienten für eine partnerschaftliche Arzt-Patienten-Beziehung und zum neuen Erwachsenenschutzrecht. Zürich: Dialog Ethik; 2013.

15 Bally K, Berchtold-Ledergerber V, Hofer G, Karger A, Kiss A, Kressig RW et al. Kommunikation im medizinischen Alltag. Ein Leitfaden für die Praxis. Basel: Schweizerische Akademie der Medizinischen Wissenschaften; 2013.
16 Charles C, Gafni A, Whelan T. Decision-making in the physician-patient encounter: revisiting the shared treatment decision-making model. Soc Sci Med. 1999;49:651-61.

17 Makoul G, Clayman ML. An integrative model of shared decision making in medical encounters. Patient Educ Couns. 2006;60:301-12.

18 Moumjid N, Gafni A, Bremond A, Carrere MO. Shared decision making in the medical encounter: are we all talking about the same thing? Med Decis Making. 2007;27:539-46.

19 Elwyn G, Frosch D, Rollnick S. Dual equipoise shared decision making: definitions for decision and behaviour support interventions. Implement Sci. 2009;4:75.

20 Frosch DL, Kaplan RM. Shared decision making in clinical medicine: past research and future directions. Am J Prev Med. 1999;17:285-94.

21 Hoffmann TC, Légaré F., Simmons MB, McNamara K, McCaffery K, Trevena LJ et al. Shared decision making: what do clinicians need to know and why should they bother? The Medical Journal of Australia. 2014;201:35-39.

22 Elwyn G, Edwards A, Kinnersley P. Shared decisionmaking in primary care: the neglected second half of the consultation. Br J Gen Pract. 1999;49:477-82.

23 Wirtz V, Cribb A, Barber N. Patient-doctor decisionmaking about treatment within the consultation a critical analysis of models. Soc Sci Med. 2006;62:116-24.

24 Loh A, Simon D. Gemeinsam entscheiden - erfolgreich behandeln? Managed Care. 2007;2:6-8.

25 Elwyn G, Frosch D, Thomson R, Joseph-Williams N, Lloyd A, Kinnersley P, et al. Shared decision making: a model for clinical practice. J Gen Intern Med. 2012;27:1361-7.

26 Scheibler F, Janssen C, Pfaff H. Shared decision making: ein Überblicksartikel über die internationale Forschungsliteratur. Sozial- und Präventivmedizin. 2003;48:11-23

27 Joseph-Williams N, Elwyn G, Edwards A. Knowledge is not power for patients: A systematic review and thematic synthesis of patient-reported barriers and facilitators to shared decision making. Patient education and counseling. 2014;94:291-309.

28 Entwistle VA, Watt IS. Patient involvement in treatment decision-making: the case for a broader conceptual framework. Patient Educ Couns. 2006;63:268-78.

20 Faller H. Shared Decision Making: Ein Ansatz zur Stärkung der Partizipation des Patienten in der Rehabilitation. Rehabilitation. 2003;42:129-35.

30 Matthias MS, Salyers MP, Frankel RM. Re-thinking shared decision-making: context matters. Patient Educ Couns. 2013;91:176-9.

31 Montori VM, Gafni A, Charles C. A shared treatment decision-making approach between patients with chronic conditions and their clinicians: the case of diabetes. Health Expectations. 2006;9:25-36.

32 BGE 117 Ib 197

33 Zimmerli U. Die Verantwortung für den Patienten im öffentlichen Spital. Schweizerische Ärztezeitung. 1991;72:612-617. 
34 Cicoria K. Beweislastverteilung und Beweiserleichterung im Arzthaftungsprozess. Jusletter. 12. April 2010.

35 Stiggelbout AM, Van der Weijden T, De Wit MP, Frosch D, Legare F, Montori VM, et al. Shared decision making: really putting patients at the centre of healthcare. Bmj. 2012;344:e256.

36 Lenz M, Buhse S, Kasper J, Kupfer R, Richter T, Muhlhauser I. Decision aids for patients. Deutsches Ärzteblatt International. 2012;109:401-8.

37 Stacey D, Légaré F, Col NF, Bennett CL, Barry MJ, Eden $\mathrm{KB}$, et al. Decision aids for people facing health treatment or screening decisions. Cochrane Database Syst Rev. 2014;1:CD001431.

38 Karnieli-Miller O, Eisikovits Z. Physician as partner or salesman? Shared decision-making in real-time encounters. Soc Sci Med. 2009;69:1-8.

39 Scheibler F, Pfaff H. Shared decision-making. Ein neues Konzept für Professionellen-Patienten-Interaktion. In: Scheibler F, Pfaff H (eds.). Shared Decision-Making. Der Patient als Partner im medizinischen Entscheidungsprozess. Weinheim: Juventa Verlag; 2003. p. 11-22.

40 Politi MC, Studts JL, Hayslip JW. Shared decision making in oncology practice: what do oncologists need to know? Oncologist. 2012;17:91-100.

41 Kryworuchko J, Hill E, Murray MA, Stacey D, Fergusson DA. Interventions for shared decisionmaking about life support in the intensive care unit: a systematic review. Worldviews Evid Based Nurs. 2013;10:3-16.

42 Körner M. Ein Modell der partizipativen Entscheidungsfindung in der medizinischen Rehabilitation. Rehabilitation. 2009;48:160-165.

43 Drake RE, Cimpean D, Torrey WC. Shared decision making in mental health: prospects for personalized medicine. Dialogues Clin Neurosci. 2009;11:455-63.

44 Elwyn G, Dehlendorf C, Epstein RM, Marrin K, White J, Frosch DL. Shared decision making and motivational interviewing: achieving patient-centered care across the spectrum of health care problems. Ann Fam Med. 2014;12:270-5.

45 Chewning B, Bylund CL, Shah B, Arora NK, Gueguen JA, Makoul G. Patient preferences for shared decisions: a systematic review. Patient Educ Couns. 2012;86:9-18.

46 Floer B, Schnee M, Bocken J, Streich W, Kunstmann W, Isfort J et al. Shared Decision Making. Gemeinsame Entscheidungsfindung aus Patientenperspektive. Dtsch Med Wochenschr. 2004;129·2343-7.

47 Hamann J, Bieber C, Elwyn G, Wartner E, Horlein E, Kissling W, et al. How do patients from eastern and western Germany compare with regard to their preferences for shared decision making? Eur J Public Health. 2011;22:469-73.
48 Couët N, Desroches S, Robitaille H, Vaillancourt H, Leblanc A, Turcotte S, et al. Assessments of the extent to which health-care providers involve patients in decision making: a systematic review of studies using the OPTION instrument. Health Expect. 2013.

49 Légaré F, Ratte S, Gravel K, Graham ID. Barriers and facilitators to implementing shared decision-making in clinical practice: update of a systematic review of health professionals' perceptions. Patient Educ Couns. 2008;73:526-35.

50 Duncan E, Best C, Hagen S. Shared decision making interventions for people with mental health conditions. Cochrane Database Syst Rev. 2010:CD007297.

51 Joosten EA, DeFuentes-Merillas L, de Weert GH, Sensky T, van der Staak CP, de Jong CA. Systematic review of the effects of shared decision-making on patient satisfaction, treatment adherence and health status. Psychother Psychosom. 2008;77:219-26.

52 Sanders AR, van Weeghel I, Vogelaar M, Verheul W, Pieters RH, de Wit NJ, et al. Effects of improved patient participation in primary care on health-related outcomes: a systematic review. Fam Pract. 2013;30:365-78.

53 Wennberg JE, Thomson PY. Time to tackle unwarranted variations in practice. BMJ. 2011;342:687-690.

54 Légaré F, Labrecque M, Cauchon M, Castel J, Turcotte S, Grimshaw J. Training family physicians in shared decision-making to reduce the overuse of antibiotics in acute respiratory infections: a cluster randomized trial. Cmaj. 2012;184:E726-34.

55 Deschênes SM, Gagnon MP, Legare F, Lapointe A, Turcotte S, Desroches S. Psychosocial factors of dietitians' intentions to adopt shared decision making behaviours: a cross-sectional survey. PLoS One. 2013;8:e64523.

56 Edwards A, Elwyn G, Hood K, Atwell C, Robling M, Houston $\mathrm{H}$, et al. Patient-based outcome results from a cluster randomized trial of shared decision making skill development and use of risk communication aids in general practice. Fam Pract. 2004;21:347-54.

57 Légaré F, Politi MC, Drolet R, Desroches S, Stacey D, Bekker H. Training health professionals in shared decision-making: an international environmental scan. Patient Educ Couns. 2012;88:159-69. 